\title{
Efecto anticorrosivo de pinturas de poliuretano aplicadas sobre acero galvanizado sin tratamiento superficial
}

\author{
Anti-corrosive effect of polyurethane paints applied on galvanized steel \\ without surface treatment
}

\author{
Hugo Romero Bonilla', Rosa Vera Aravena², Felipe Redrovan Pesantez ${ }^{3}$, Thalia Vera Infante ${ }^{4}$, \\ Vanessa Fernández Vélez ${ }^{5}$
}

Recibido: Junio 2019 - Aprobado: Julio 2019

\begin{abstract}
RESUMEN
El objetivo de esta investigación fue demostrar la efectividad de las pinturas de poliuretano para la protección contra la corrosión del acero galvanizado. La metodología consistió en realizar análisis electroquímico mediante curvas de polarización y pruebas de corrosión acelerada (cámara de niebla salina) del comportamiento de los sistemas de protección dúplex y galvanizado en $\mathrm{NaCl}$ al 3,5\%. Además, el análisis MEB (microscopía electrónica de barrido) se llevó a cabo con un análisis EDAX (espectroscopia de rayos $\mathrm{X}$ de energía dispersiva) observando la morfología y la composición de los productos de corrosión. Según las curvas de polarización, hay diferencias en cada sistema galvanizado y dúplex en el medio estudiado. Los sistemas dúplex tienen un potencial de corrosión más positivo que los potenciales de las muestras de acero galvanizado en cámara de niebla salina (-950mv; -1070mv de acero dúplex y -1065mv; 1080mv de acero dúplex original). Las velocidades de corrosión para el acero galvanizado y dúplex encontradas fueron de 4.8528 × 10-4 1,8045 x 10-5 cm/año respectivamente en la cámara de niebla salina. Estas condiciones de exposición dejan claro que el sistema dúplex, se comporta más protector que el sistema galvanizado. A partir del análisis EDAX se encontró que, los productos de corrosión que se esperaría encontrar para el caso del óxido formado en acero galvanizado después de la experimentación en la cámara de niebla salina sería una mezcla de sales de zinc y hierro como por ejemplo zincita, hidrocincita, simonkoleita, y hematita para el caso del hierro, como también sales solubles (cloruros) de estos metales, así como, óxidos de hierro y zinc, además de zinc y hierro en su estado metálico.
\end{abstract}

Palabras clave: Corrosión; acero galvanizado; pintura poliuretano; acero dúplex.

\begin{abstract}
The objective of this research was to demonstrate the effectiveness of polyurethane paints for the protection against corrosion of galvanized steel. The methodology consisted of performing electrochemical analysis by means of polarization curves and accelerated corrosion tests (salt spray chamber) of the behavior of the duplex and galvanized protection systems in $3.5 \% \mathrm{NaCl}$. In addition, the SEM analysis (scanning electron microscopy) was carried out with an EDAX analysis (X-ray spectroscopy of dispersive energy) observing the morphology and the composition of the corrosion products. According to the polarization curves, there are differences in each galvanized and duplex system in the studied medium. The duplex systems have a more positive corrosion potential than the potentials of the galvanized steel samples in the salt fog chamber $(-950 \mathrm{mv},-1070 \mathrm{mv}$ of duplex steel and $-1065 \mathrm{mv}, 1080 \mathrm{mv}$ of original duplex steel). The corrosion rates for the galvanized and duplex steel found were $4.8528 \times 10-41.8045 \times 10-5 \mathrm{~cm} /$ year respectively in the salt spray chamber. These exposure conditions make it clear that the duplex system behaves more protective than the galvanized
\end{abstract}

\footnotetext{
1 Jefe del Laboratorio de Electroanalítica y Bioenergía, Unidad Académica de Ciencias Químicas y de la Salud, Universidad Técnica de Machala, Ecuador, Av. Panamericana km. 5 1/2 Via a Pasaje, código postal 070102. E-mail: hromero@utmachala.edu.ec; ORCID: 0000-0002-7846-0512

2 Investigadora, Laboratorio de Corrosión, Instituto de Química, Pontificia Universidad Católica de Valparaíso, Av. Brasil 2950, código postal 070102, Laboratorio de corrosión, Pontificia Universidad Católica de Valparaíso, Universidad Avenue 330 - Curauma, Valparaíso, Región de Valparaíso, Chile. E-mail: rosa.vera@pucv.cl; ORCID: 0000-0001-8801-9758

3 Investigador, carrera de Ingeniería en Alimentos, Unidad Académica de Ciencias Químicas y de la Salud, Universidad Técnica de Machala. E-mail: fredrovan2@ utmachala.edu.ec; ORCID: 0000-0001-9157-4760. Autor de correspondencia.

4 Investigadora, Laboratorio de Electroanalítica y Bioenergía, Unidad Académica de Ciencias Químicas y de la Salud, Universidad Técnica de Machala, Ecuador. E-mail: tgverai_est@utmachala.edu.ec,vera2cthalia@gmail.com; ORCID: Código 0000-0002-7076-2639

5 Investigadora, Laboratorio de Electroanalítica y Bioenergía, Unidad Académica de Ciencias Químicas y de la Salud, Universidad Técnica de Machala. E-mail:: cvfernandez_est@utmachala.edu.ec 
system. From the EDAX analysis it was found that the corrosion products that would be expected to be found in the case of the oxide formed in galvanized steel after experimentation in the salt fog chamber would be a mixture of zinc and iron salts such as zincite, hydrocincite, simonkoleite, and hematite for iron, as well as soluble salts (chlorides) of these metals, as well as iron and zinc oxides, as well as zinc and iron in their metallic state.

Keywords: Corrosion; galvanized steel; polyurethane paint; duplex steel.

\section{INTRODUCCIÓN}

Los aceros son muy susceptibles a la corrosión, proceso electroquímico que causa un deterioro generalmente uniforme (Bermúdez et al, 2012). En la mayor parte de los procesos industriales interviene el contacto con fluidos de diferente naturaleza (Bergaño, 2015). Es así como las piezas expuestas a la acción de estos pueden presentar procesos de corrosión por erosión, lo que disminuye de manera acelerada su vida útil (Aperador et al, 2013).

El proceso de corrosión, es muy complejo, debido a que involucra fenómenos tales como el flujo de iones, reacciones electroquímicas, craqueo y plasticidad. A pesar de que se ha estudiado extensamente, sin embargo, aún existen una serie de cuestiones que no están claras (Papakonstantinou \& Shinozuka, 2013).

El hierro al estar expuestos a la acción del clima origina el fenómeno de oxidación en su superficie, formando principalmente óxido de hierro, hidróxido y sales (Almenara et al, 2015) y (Bardal, 2007). Por otro lado, el uso de acero inoxidable dúplex en la fabricación de componentes, especialmente en la industria química, petrolera y petroquímica, está en auge debido a su alta resistencia a la corrosión y sus buenas propiedades mecánicas (Bermúdez \& González, 2012). Actualmente, las investigaciones sobre acero inoxidable dúplex y acero galvanizados han ido incrementándose(Pérez, 2002) .

El acero galvanizado consiste en la inmersión del acero en zinc-fundido a presión a $450^{\circ} \mathrm{C}$. Durante la inmersión, la difusión del zinc se origina en la superficie del acero, lo que da lugar a la formación de diferentes capas de aleaciones de zinc-hierro sobre el mismo (Vilche et al., 2002). El zinc es un metal que da lugar a la formación de hidróxido de zinc, de acuerdo con las siguientes reacciones en presencia de oxígeno y humedad:

$\begin{array}{lll}\text { Reacción anódica: } & 2 \mathrm{Zn}^{0} \quad 2 \mathrm{Zn}^{++}+4 e^{-} \\ \text {Reacción catódica: } & \mathrm{O}_{2}+2 \mathrm{H}_{2} \mathrm{O}+4 e-4 \mathrm{OH}^{-} \\ \text {Reacción global: } & 2 \mathrm{Zn}^{0}+2 \mathrm{H}_{2} \mathrm{O}+\mathrm{O}_{2} 2 \mathrm{Zn}(\mathrm{OH})_{2}\end{array}$

El acero galvanizado es el más utilizado en estructuras, debido a su buena resistencia a la corrosión atmosférica y su costo relativamente bajo (Worsley et al., 2001), especialmente cuando las atmósferas son de agresividad moderada (Schmiedl \& Guevara, 2017). En otras circunstancias, el recubrimiento orgánico es más recomendable, ventajosamente se dispone de pinturas anticorrosivas, que son muy efectivas como efecto barrera contra la corrosión electroquímica (Rani \& Basu, 2011) y (Guerrero, 2016). Existen pocas fuentes en las que se haya investigado, desarrollado y probado cómo controlar el fenómeno de la corrosión mediante recubrimientos con un espesor de fracciones de milímetro; aunque todavía queda mucho por investigar, se han logrado avances en la reducción de los costos de conservación deeste material mediante recubrimientos(Kaesche, 2012). La industria de la pintura ha desarrollado varias estrategias específicas de recubrimiento para proteger el acero de la corrosión (Vera \& Cañas, 2005). Además, determinar el tiempo de falla y el tipo de degradación específica de las pinturas en ciertos entornos es uno de los principales desafíos de los investigadores y usuarios de los recubrimientos orgánicos (Romero, 2014).

Durante años, los recubrimientos de polímeros, barnices y pinturas de poliuretano (PU) han adquirido una creciente importancia en el campo de la protección contra la corrosión (Balgude \& Sabnis, 2012). Estas pinturas, desde hace algunos años, han sido introducidas como productos "top" en el campo de los recubrimientos de materiales (Arrasco., 2003). La adaptación a la protección contra la corrosión de los sustratos metálicos es de suma importancia para garantizar la fiabilidad y el rendimiento de las piezas recubiertas a largo plazo, así como el valor de los materiales recubiertos (Aguilar, 2015). Su creciente uso en la protección contra la corrosión del hierro y el acero se debe principalmente a las propiedades específicas del aglomerante, que en comparación con los sistemas tradicionales anticorrosivos, permiten un pintado más racional y seguro, así como una vida más larga de los recubrimientos (Guerrero, 2016). Este artículo tiene como objetivo demostrar la efectividad de las pinturas de poliuretano para la protección contra la corrosión, y cómo se aplican a las superficies de acero galvanizado con un tratamiento de superficie mínimo, que implica solo un desengrase de la superficie con un detergente industrial, y, mediante análisis electroquímico y las pruebas de corrosión acelerada evaluar el comportamiento de los sistemas de protección dúplex y galvanizado a medios agresivos (Pérez et al., 2002).

\section{MÉTODOS}

En cuanto al recubrimiento dúplex aplicado al acero galvanizado $(\mathrm{Fe}+\mathrm{Zn})$, se utilizó una pintura de poliuretano (PU) DCI-324-E blanca, utilizando un detergente neutro (DSA-010-I) y un 20\% de solvente industrial (DTI-005-I) para desengrasar la superficie del metal. Las muestras de acero galvanizado se cortaron previamente para posteriormente ser desengrasadas con detergente utilizando paños abrasivos de tipo Scotch Brite, y continuar con el pintado. Todo este procedimiento se llevó a cabo de acuerdo con las 
instrucciones del proveedor. A diferencia de las muestras de acero galvanizado que no fueron pintadas, se realizó un tratamiento mediante un desengrasado de la superficie con acetona. El propósito de este procedimiento fue comparar los resultados hasta que uno de los sistemas de protección falle. Se realizaron pruebas de corrosión acelerada en una cámara de niebla salina, que simularon un ambiente marino. Para esto, las muestras de acero galvanizado y dúplex fueron cortadas de $10 \times 10 \mathrm{~cm}^{2}$. La comparación visual de las muestras tomadas en la cámara de niebla salina se realizó de acuerdo con la norma ASTM B - 117 .

Para determinar el potencial de corrosión se utilizó un potenciostato-galvanostato modelo GSEC V2.0 conectado a una PC. Las muestras se midieron durante 40 días en $\mathrm{NaCl}$ al $3,5 \%$ como medio corrosivo. También se obtuvieron curvas de potencial a circuito abierto en función del tiempo y curvas de polarización para determinar las corrientes de corrosión.
Para caracterizar los productos de corrosión se utilizó microscopía electrónica de barrido (MEB) con analizador de rayos $\mathrm{X}$ mediante energía dispersiva (EDAX) y difracción de rayos $\mathrm{X}$ que proporcionaron información sobre su morfología y porcentajes de elementos presentes.

\section{RESULTADOS}

En la Figura 1 se observa una microfotografía transversal de una muestra de acero Duplex (Fe $+\mathrm{Zn}$ pintado con PU) que no fue expuesta a ningún medio agresivo. En ella se pueden observar las distintas capas que constituyen el sistema protector del metal base.

Por otro lado, la Figura 2 muestra la variación del potencial a circuito abierto enfunción del tiempo (40 días) para los dos sistemas (duplex y galvanizado) en $\mathrm{NaCl}$

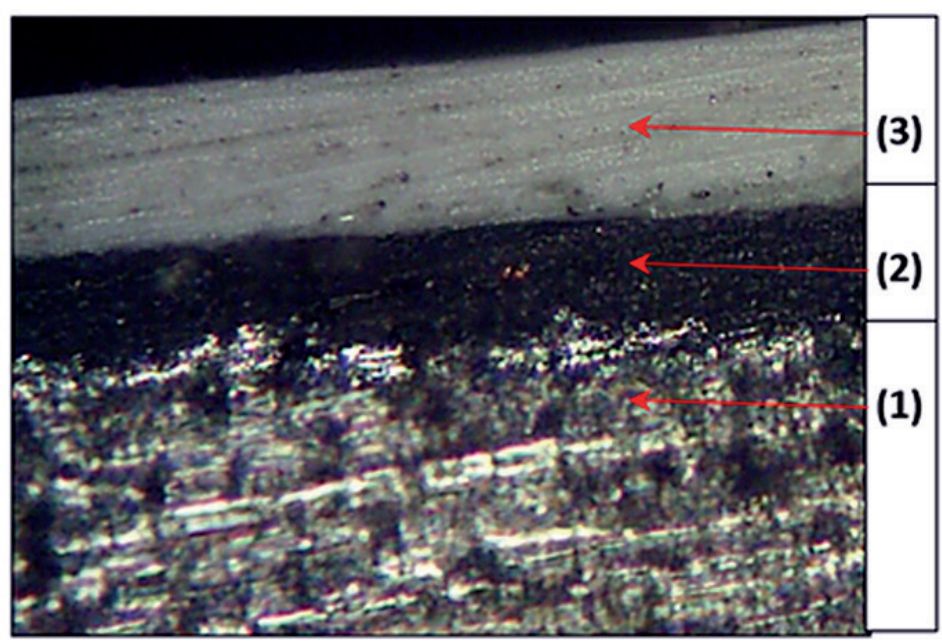

Figura 1. Microfotografía de acero galvanizado pintado sin exposición a medios agresivos (x 500) (1) Fe; (2) Zn; (3) Pintura.

Fuente: Elaboración propia

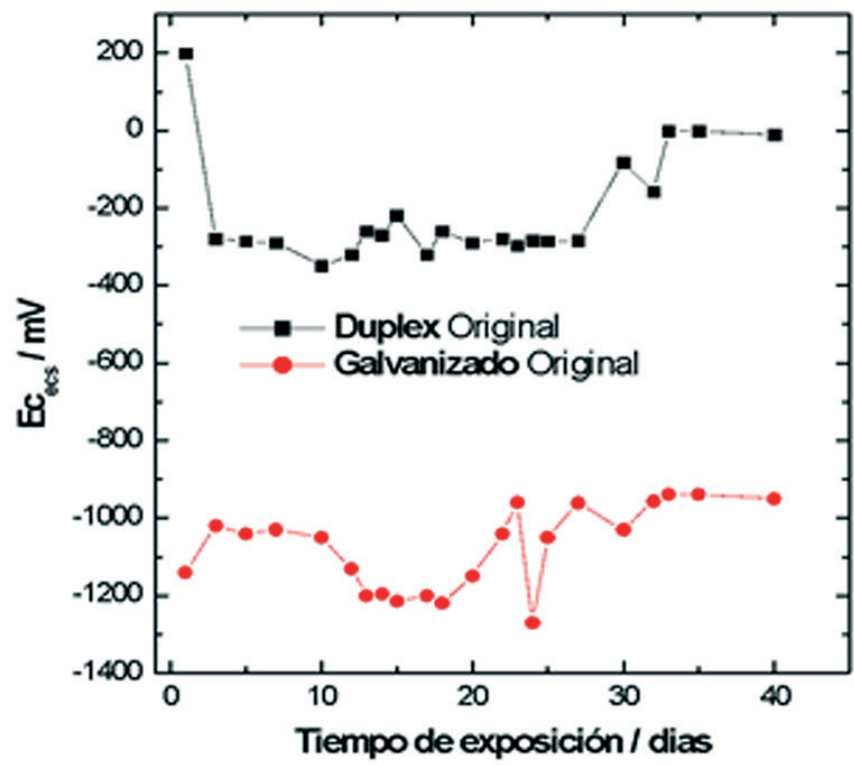

Figura 2. Potencial a circuito abierto para los sistemas duplex y galvanizado en función del tiempo en $\mathrm{NaCl} 3.5 \%$.

Fuente: Elaboración propia 
$3.5 \%$, donde es evidente que, el comportamiento del sistema duplex en un comienzo es inestable. Esto, debido principalmente al efecto que tiene la morfología de los poros del recubrimiento al intentar estabilizar la diferencia de potencial. La disminución brusca del potencial del sistema se debe a que el electrolito aún no ha alcanzado la interfase metal/pintura. Una vez alcanzada ésta, la diferencia de potencial en el sistema duplex se estabiliza pasado los 30 días de exposición a la solución salina, alrededor de los $-10 \mathrm{mV}$. Para el caso del sistema galvanizado sin pintura en $\mathrm{NaCl}$, el potencial alcanza la estabilidad mucho más rápido, pero al igual que el sistema duplex en un comienzo muestra variaciones que, con el transcurso del tiempo se hace más constante. También se observa una estabilidad después de los 30 días de exposición, pero alrededor de los $-950 \mathrm{mV}$. Este desplazamiento del potencial hacia valores más negativos aumenta la posibilidad de corrosión del acero galvanizado.

Los potenciales a circuito abierto de las muestras metálicas que fueron sometidas al ambiente de la Cámara de Niebla Salina se presentan en la Figura 3. En la de acero galvanizado se observó un producto de corrosión color rojo que se podría atribuir a la hematita $\left(\mathrm{Fe}_{2} \mathrm{O}_{3}\right)$. La muestra de acero duplex presentó un producto de corrosión blanco que estaría relacionado con las sales de cinc formadas sobre la superficie metálica como la cincita $(\mathrm{ZnO})$ y $\mathrm{Zn}(\mathrm{OH})_{2}$.

\section{DISCUSIÓN}

Se puede observar que para el caso del acero galvanizado duplex que no presenta producto de corrosión rojo (línea azul) se comporta más estable con respecto al acero galvanizado original, manteniendo en el transcurso del tiempo una estabilidad del potencial (cercano a los $-1000 \mathrm{mV}$ como promedio). Esto se traduce como una pasivación en la superficie del metal que tiene relación con los productos de corrosión del cinc. Sin embargo, en esta misma figura para las muestras que presentaron producto de corrosión rojo (línea verde) si se observa un cambio de comportamiento debido principalmente a su potencial que aumenta en aproximadamente en $200 \mathrm{mV}$ con respecto al acero galvanizado sin pintura. Debido a que el hierro comenzó a ser atacado, lo cual es concordante, ya que, los potenciales de corrosión o de equilibrio que generalmente presenta este metal se encuentran alrededor de los $-700 \mathrm{mV}$. Para el caso de la muestra de galvanizado que sufre este ataque, el potencial comienza a estabilizarse alrededor de los $-730 \mathrm{mV}$ después de los 25 días de exposición.

En lo referente a los resultados de las curvas de polarización, las diferencias de cada sistema galvanizado y dúplex en $\mathrm{NaCl}$ al 3,5\% y cámara de niebla salina (después de 14 días) se presentan en la Figura 4. Los sistemas dúplex tienen un potencial de corrosión (Ec) más positivo. El acero dúplex en la cámara de niebla salina (azul) presentó un Ec de $-950 \mathrm{mV}$ mientras que, para el acero dúplex en $\mathrm{NaCl}$ (negro) el Ec fue de $-1065 \mathrm{mV}$. Por su parte los potenciales de las muestras de acero galvanizado fueron: -1070 mV en la cámara de niebla salina (rojo) y -1080 $\mathrm{mV}$ para el galvanizado en $\mathrm{NaCl}$ (verde) respectivamente. El comportamiento más protector del acero duplex en comparación con otros sistemas se debe a que al inicio del experimento el electrolito no tiene contacto con el metal que forma la interfase con la película protectora de pintura, pero una vez alcanzado, el potencial se comporta más estable después de los 30 días de exposición. Aquí,

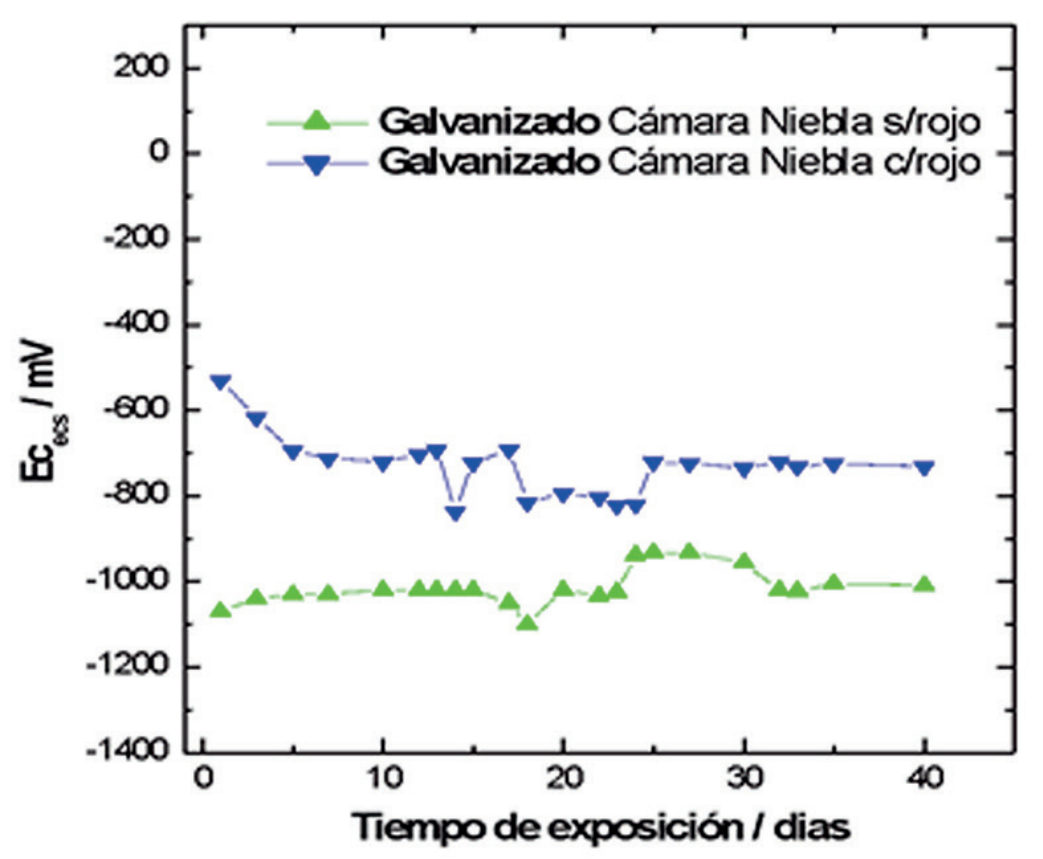

Figura 3. Potencial a circuito abierto para muestras de acero galvanizado extraídas de Cámara de Niebla Salina, en función del tiempo en $\mathrm{NaCl} 3.5 \%$; galvanizado duplex (azul): s/rojo (sin producto de corrosión rojo); solo galvanizado (verde): c/rojo (con producto de corrosión rojo).

Fuente: Elaboración propia 
los productos de corrosión actúan como un "tapón" en los poros de la película de polímero que se traduce en una doble protección contra la corrosión (Briceño \& Guevara, 2017).

En lo referente a las corrientes de corrosión, para el sistema duplex en $\mathrm{NaCl}$, en la Figura 4 se observa que la corriente tiene valores del orden de 10-11 [A x cm-2]. Por su parte, en el sistema duplex expuesto a Cámara de Niebla Salina la corriente disminuye cercano a los 10-8 [A x cm2], pero el potencial aumenta cerca de los -950 [mV]. Este comportamiento indica que, en la cámara de niebla salina, la superficie del polímero se ve afectado aumentando la velocidad de corrosión, pero al mismo tiempo los productos de corrosión que se forman aumentan el potencial hacia valores positivos mejorando el comportamiento frente a los sistemas de galvanizado. En la tabla 1 se pueden observar las corrientes y velocidades de corrosióncalculadas a partirde esta figura. También se observa que la corriente anódica comienza a estabilizarse cerca de los $-800[\mathrm{mV}]$ en adelante, esto puede deber su explicación a que una vez que el electrolito ha alcanzado el sustrato metálico los productos de corrosión que comienzan a aparecer forman una doble capa protectora con la película de pintura, pasivando la superficie metálica. En este punto es preciso explicar que para obtener datos reproducibles, fue necesario esperar un tiempo para que el sistema alcance un equilibrio (dos semanas).

Por otra parte, la corriente catódica nos muestra una disolución activa del metal más lenta que se demuestra en los cálculos de velocidades de corrosión (Tabla 1), y que debe su explicación al igual que en las corrientes anódicas al paso más lento del electrolito hacia la interfase del sistema duplex por la morfología que presenta la matriz polimérica.

La pasivación de la superficie del galvanizado que estuvo expuesto a cámara de niebla se observa después de los $-800[\mathrm{mV}]$, en cambio para la muestra de galvanizado en $\mathrm{NaCl}$ no se observa una pasivación. De igual forma para las corrientes catódicas se observan diferencias que se traducen en las velocidades de corrosión (Tabla 1), siendo

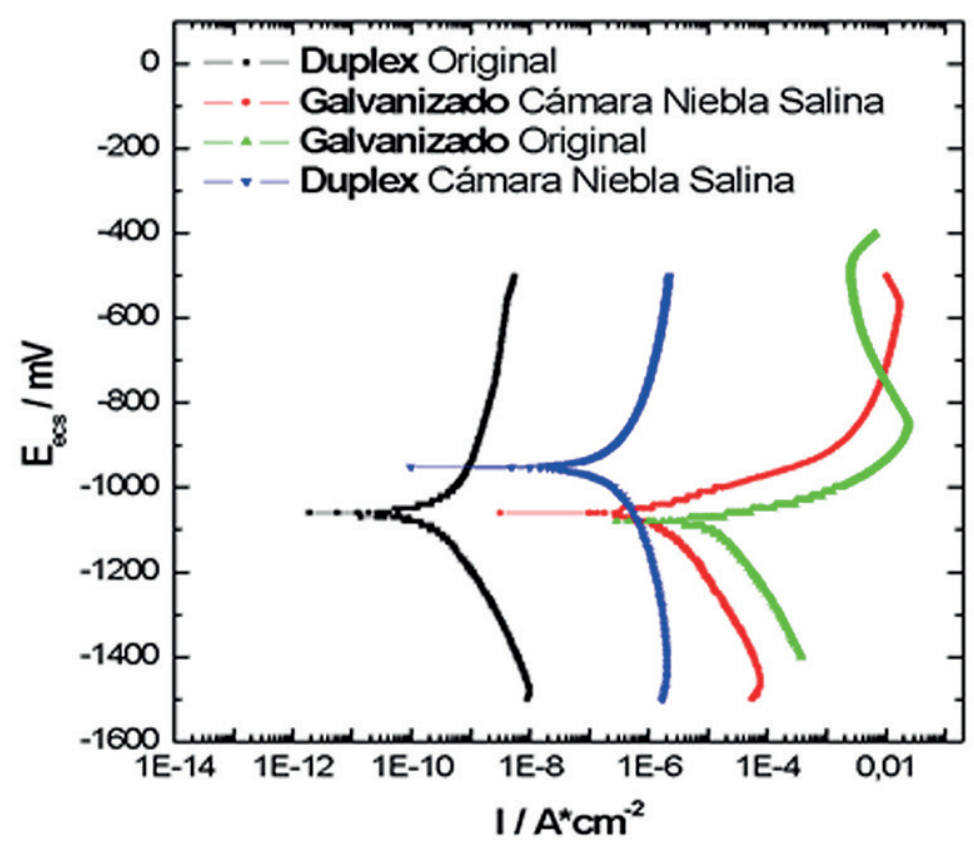

Figura 4. Curvas de polarización para los sistemas duplex, galvanizado y galvanizado en cámara de niebla salina (336 horas), en $\mathrm{NaCl} 3,5 \%$.

Fuente: Elaboración propia

Tabla 1. Determinación de velocidad de corrosión para acero galvanizado en $\mathrm{NaCl}$, galvanizado en Cámara de Niebla Salina (después de 14 días) y duplex en $\mathrm{NaCl} 3.5 \%$ y Cámara de Niebla Salina (después de 14 días)

\begin{tabular}{|c|c|c|c|c|}
\hline Muestra & Galvanizado en $\mathrm{NaCl}$ & Duplex en $\mathrm{NaCl}$ & $\begin{array}{l}\text { Galvanizado en Cámara de } \\
\text { Niebla Salina }\end{array}$ & $\begin{array}{l}\text { Duplex en Cámara de } \\
\text { Niebla Salina }\end{array}$ \\
\hline Solución & $\mathrm{NaCl} 3,5 \%$ & $\mathrm{NaCl} 3,5 \%$ & $\mathrm{NaCl} 3,5 \%$ & $\mathrm{NaCl} 3,5 \%$ \\
\hline $\mathrm{Ic}\left[\mathrm{A} / \mathrm{cm}^{2}\right]$ & $9,9090 \times 10^{-6}$ & $8,3835 \times 10^{-11}$ & $3,2434 \times 10^{-7}$ & $1,2517 \times 10^{-8}$ \\
\hline Peq. [gr. /eq.] & 32,69 & 32,69 & 32,69 & 32,69 \\
\hline (R) [c/eq.] & 96500 & 96500 & 96500 & 96500 \\
\hline$\rho\left[\mathrm{gr} / \mathrm{cm}^{3}\right]$ & 7,14 & 7,14 & 7,14 & 7,14 \\
\hline Vc [cm/año] & 0,0148 & $1,2543 \times 10^{-7}$ & $4,8528 \times 10^{-4}$ & $1,8045 \times 10^{-5}$ \\
\hline
\end{tabular}

Fuente: Elaboración propia 
más lenta para el galvanizado que estuvo expuesto a cámara de niebla salina que para el galvanizado en $\mathrm{NaCl}$ ya que se forma una barrera sobre la superficie del metal.

En estas condiciones de exposición es evidente que el sistema de acero duplex se comporta mucho más protector que el sistema de acero galvanizado, por lo cual la cinética que presentó éste al paso de electrolito es más efectivo. Así también se observa una variación de la corriente entre ellas, alcanzando cuatro y cinco órdenes de magnitud de diferencia.

De esta Tabla se obtiene información que confirma lo analizado anteriormente, donde los productos de corrosión que se están formando sobre la superficie del metal comienzan a reducir la velocidad de corrosión. El comportamiento del acero duplex frente a estas condiciones es inmejorable hasta en 5 décadas en comparación al galvanizado y 2 décadas al compararlos con los galvanizados que estuvieron expuestos a Cámara de Niebla Salina.

Los análisis desarrollados por MEB y EDAX entregan importante información acerca de las capas que constituyen los sistemas de Galvanizado y Duplex, y de los productos de corrosión en forma porcentual de cada elemento.

En la Figura 5 se observa una microfotografía del acero duplex que fue expuesto a la cámara de niebla salina.

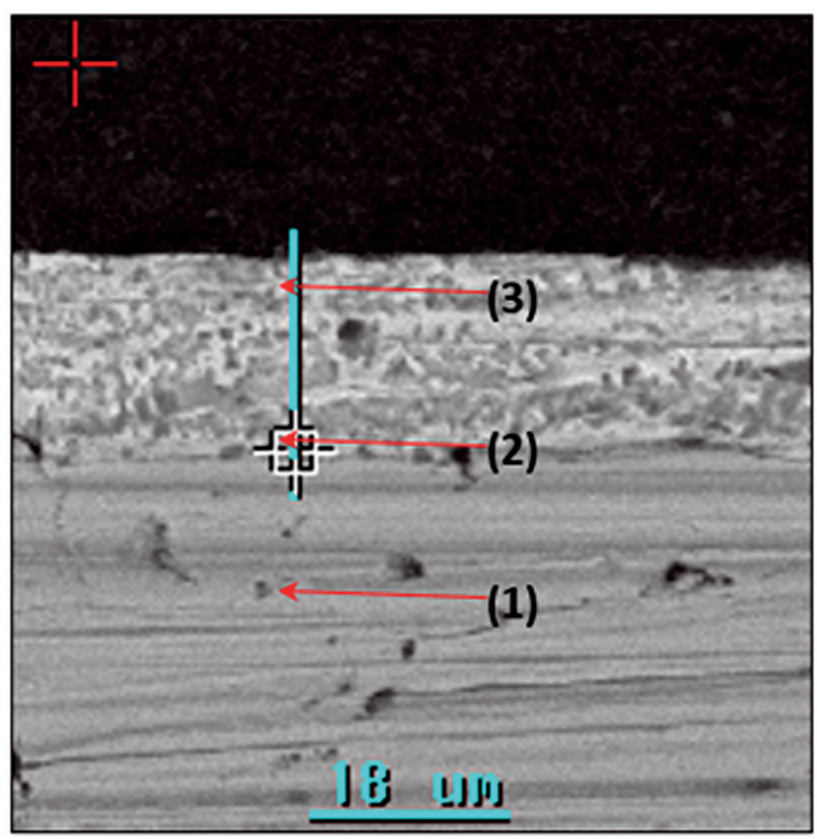

Figura 5. Microfotografía de un sistema Duplex (x 2000) (1) Fe; (2)

Zn; (3) Pintura, antes de exposición en Cámara de Niebla(336 horas), donde $18 \mu \mathrm{m}=3,3 \mathrm{~cm}$.

Fuente: Elaboración propia

Por otro lado, en la Figura 6 (zona 4 y 3 ) se observa una microfotografía de la superficie del galvanizado expuesta a la cámara de niebla salina, donde es evidente un producto de corrosión con crecimiento continuo.

Se realizó un análisis EDAX (Figura 7) a las zonas 2, 3 y 4 de la Figura 6, lo cual permitió establecer posibles productos de corrosión generados durante el proceso corrosivo.De esta manera los productos de corrosión que se esperaría encontrar para el caso de la zona 4 (Figura 7a) sería una mezcla de sales de cinc y hierro como por ejemplo cincita, hidrocincita, simonkoleita, y hematita para el caso del hierro, como también sales solubles (cloruros) de estos metales. Para la zona 2 (Figura 7b) los productos de corrosión serían principalmente a óxidos de hierro y cinc, además de cinc y hierro en su estado metálico. De la zona 3 (Figura 7c) se esperaría encontrar producto de corrosión en base a óxido de zinc (cincita).

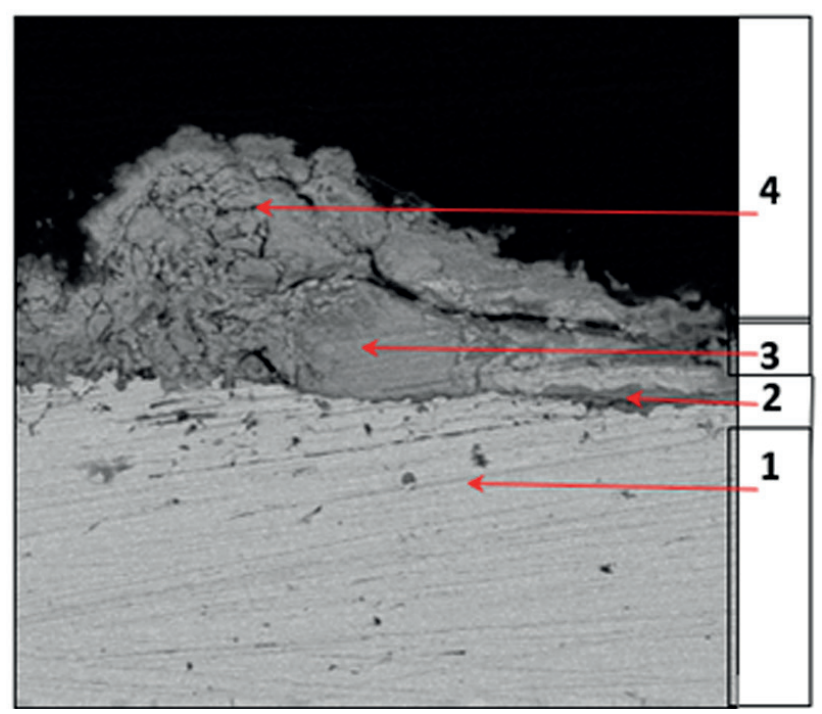

Figura 6. Microfotografía de acero galvanizado desnudo después de 336 horas de exposición a Cámara de Niebla Salina(x 2000) (1) Fe; (2)

$$
\begin{gathered}
\mathrm{Fe}_{2} \mathrm{O}_{3 ;} \text { (3) } \mathrm{ZnO} \text {; (4) } \mathrm{ZnCl}_{2} \times 4 \mathrm{Zn}(\mathrm{OH})_{2} \text {. } \\
\text { Fuente: Elaboración propia }
\end{gathered}
$$

En este análisis EDAX realizados para los productos de corrosión, muestra al zinc como principal constituyente, por esto se esperaría que la mayoría del producto de corrosión son sales u óxidos de cinc $\left(\mathrm{Zn}(\mathrm{OH})_{2}, \mathrm{ZnO}\right)$, que posiblemente que se encuentren hidratados. Esto no significa la exclusión de producto de corrosión de hierro ya que de igual manera lo encontramos, eso sí en un porcentaje menor $\left(\mathrm{Fe}_{2} \mathrm{O}_{3}\right)$ respecto al total. Según la morfología de estos productos de corrosión, además de los EDAX desarrollados en estudios anteriores, es posible encontrar una mezcla de productos de corrosión que corresponderían a: $\mathrm{ZnO}$ (cincita), $\mathrm{Zn}_{5}(\mathrm{CO} 3)_{2}(\mathrm{OH})_{6}$ (hidrocincita), $\mathrm{Zn}_{5}$ $(\mathrm{OH})_{8} \mathrm{Cl}_{2} \times \mathrm{H}_{2} \mathrm{O}$ (simonkoleita). Esto es interpretado con los porcentajes extraídos de las tablas que se adjuntan a los análisis EDAX para cada Figura 7.

Con respecto a la morfología que presentan los productos de corrosión fue necesario realizar una microscopia superficial. En las Figura 8 se muestra el producto de corrosión del acero galvanizado después de 336 horas en cámara de Niebla Salina.

En consecuencia, esta metodología entrega mucha información para dilucidar un poco más acerca de la morfología, y de cómo se podrían encontrar los productos de corrosión. 


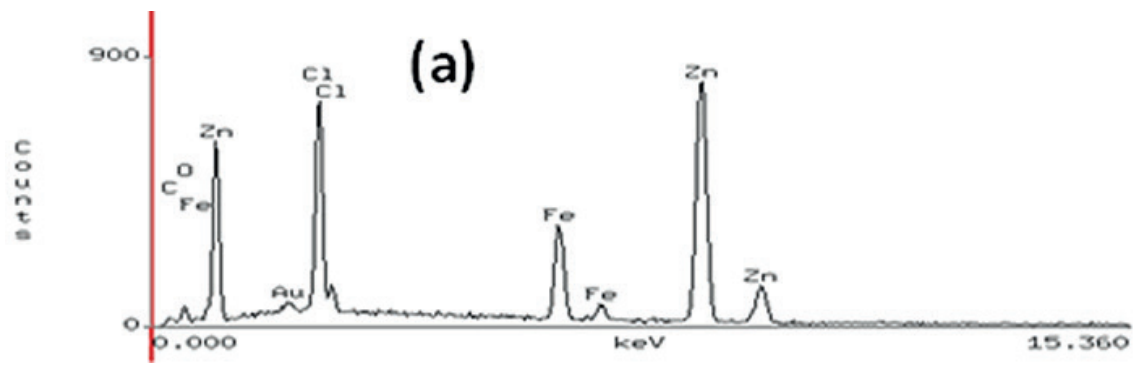

\begin{tabular}{|l|c|}
\hline Elementos & Elemento [\%] \\
\hline $\mathrm{O}$ & 6,02 \\
\hline $\mathrm{Cl}$ & 14,57 \\
\hline $\mathrm{Fe}$ & 12,40 \\
\hline $\mathrm{Zn}$ & 67,00 \\
\hline Total & 100 \\
\hline
\end{tabular}

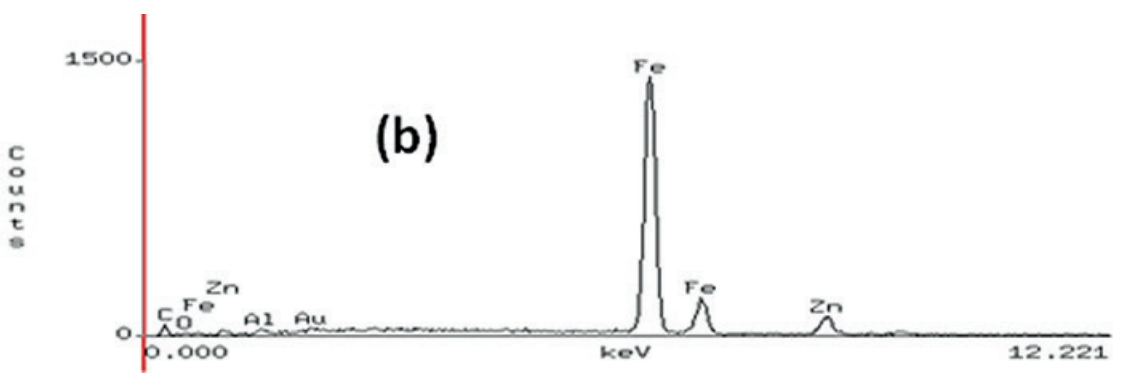

\begin{tabular}{|l|c|}
\hline Elementos & Elemento [\%] \\
\hline $\mathrm{O}$ & 1,14 \\
\hline $\mathrm{Fe}$ & 85,98 \\
\hline $\mathrm{Zn}$ & 12,89 \\
\hline Total & 100 \\
\hline
\end{tabular}

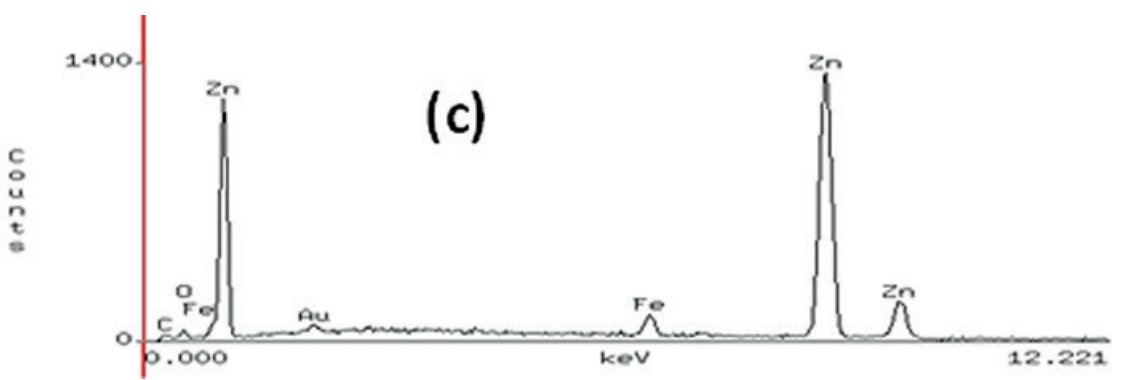

\begin{tabular}{|l|c|}
\hline Elementos & Elemento [\%] \\
\hline $\mathrm{O}$ & 3,20 \\
\hline $\mathrm{Fe}$ & 3,40 \\
\hline $\mathrm{Zn}$ & 93,41 \\
\hline Total & 100 \\
\hline
\end{tabular}

Figura 7. MEB de las tres zonas del producto de corrosión de la Figura 6, (a) zona 4; (b) zona 2; (c) zona 3.
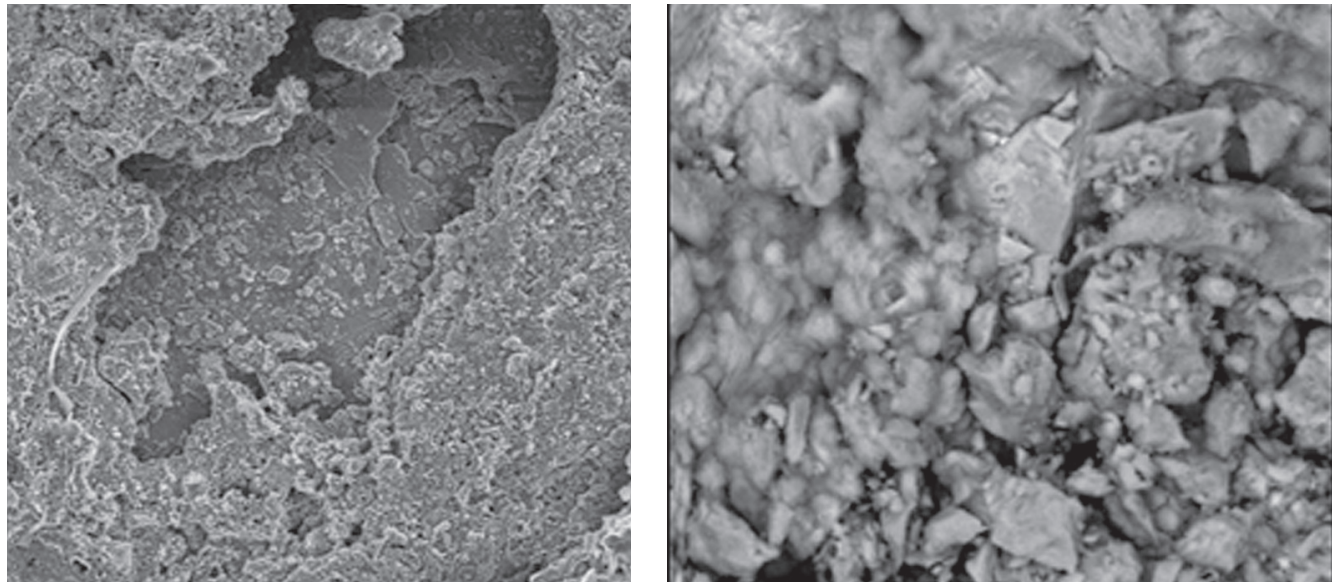

Figura 8. Microfotografía de producto de corrosión del acero galvanizado después de 336 horas en Cámara de Niebla Salina (corte superficial) (x 500).

\section{CONCLUSIONES}

Los sistemas dúplex tienen un potencial de corrosión más positivo que los potenciales de las muestras de acero galvanizado en cámara de niebla salina $(-950 \mathrm{mv} ;-1070 \mathrm{mv}$ de acero dúplex y $-1065 \mathrm{mv} ; 1080 \mathrm{mv}$ de acero dúplex original). Las velocidades de corrosión para el acero galvanizado $\mathrm{y}$ dúplex encontradas fueron de $4.8528 \mathrm{x}$ $10^{-4} 1,8045 \times 10^{-5} \mathrm{~cm} /$ año respectivamente en la cámara de niebla salina. Estas condiciones de exposición dejan claro que el sistema dúplex, se comporta más protector que el sistema galvanizado. A partir del análisis EDAX se encontró que, los productos de corrosión que se esperaría encontrar para el caso del óxido formado en acero galvanizado después de la experimentación en la cámara de niebla salina sería una mezcla de sales de zinc y hierro como por ejemplo zincita, hidrocincita, simonkoleita, y hematita para el caso del hierro, como también sales solubles (cloruros) de estos metales, así como, óxidos de hierro y zinc, además de zinc y hierro en su estado metálico. 
Cabe señalar que, en comparación con el sistema galvanizado desnudo, el sistema duplex retardó el proceso destructivo de la muestra lo que significa una ganancia de tiempo importante en casos extremos en una Industria que cuente con este sistema de protección.

Los recubrimientos de pintura a base de poliuretano, no solo protegen y aumentan la resistencia contra los procesos destructivos de forma mucho más efectiva y duradera que el acero galvanizado por sí solo, sino que también lleva intrínsecamente un ahorro en dinero, lo que produciría un gran impacto y beneficios para la empresa, industria y trabajadores que utilicen el sistema duplex como medio de protección frente a la corrosión.

\section{REFERENCIAS BIBLIOGRAFICAS}

Almenara, M. D., Rosales, A., Vidal, J., Muñoz, P., y Payares, M. (2015). Influencia de soldaduras $S A W$ sobre la resistencia a la corrosión de aceros duplex SAF 2205. Suplemento de la Revista Latinoamericana de Metalurgia y Materiales 2009; S1 (1): 289-296. Universidad Simón Bolívar (Venezuela). Recuperado de http://www.rlmm.org/archivos/S01/N1/ RLMMArt-09S01N1-p289.pdf

Operador, W., Bautista, J., y Betancur, J. (2013). Resistencia al desgaste erosivo-corrosivo de aceros austeníticos fermanal (erosive-corrosive wear resistance of fermanal austenitic steels). Revista EIA, 9(18), 49-59. Recuperado de https:// revistabme.eia.edu.co/revistas/index.php/reveia/article/ view/259

Arrasco Mejía, N. E. (2003). Reducción del nivel de rechazos en una planta de pinturas (tesis de grado). Facultad de Ingeniería Química y Textil. Universidad Nacional de Ingeniería. Recuperado de http://cybertesis.uni.edu.pe/ handle/uni/8678

Balgude, D., \& Sabnis, A. (2012). Sol-gel derived hybrid coatings as an environment friendly surface treatment for corrosion protection of metals and their alloys. Journal of Sol-Gel Science and Technology, 64(1), 124-134. https:// doi.org/10.1007/s10971-012-2838-z

Bardal, E. (2007). Corrosion and Protection. Springer Science \& Business Media, 14 jun. 2007 - 315 páginas. Recuperado de http://bit.ly/2EuStE0

Bergaño Aguilar, E. A. (2015). Recubrimientos necesarios en construcciones civiles con estructuras de acero para evitar la presencia de corrosión y mantenimientos estructurales. Programa de Ingeniería Civil, Facultad de Ingeniería, Universidad Católica de Colombia Bogotá D.C., Colombia. Recuperado de https://repository.ucatolica.edu.co/ bitstream/10983/2520/3/Articulo.pdf

Bermúdez-Castañeda, Á., Castaño-González, J.G., y Echeverría Echeverría, F. (2012). Deterioro de recubrimientos orgánicos: Principales modelos y métodos de cuantifica. Ingeniería y Desarrollo, 30(2), 261-283. Recuperado de http://www. scielo.org.co/scielo.php?script=sci_arttext\&pid=S0122$34612012000200008 \& \operatorname{lng}=$ en\&tlng $=$ es.
Kaesche, H. (2012). Corrosion of Metals: Physicochemical Principles and Current Problems. University ErlangenNurnberg. Institute for Materials Sciences. Germany, pp. 594. Springer Science \& Business Media. Recuperado de http://bit.ly/2s1ySIV

Papakonstantinou, K. G., \& Shinozuka, M. (2013). Probabilistic model for steel corrosion in reinforced concrete structures of large dimensions considering crack effects. Engineering Structures, 57, 306-326. https://doi.org/10.1016/j. engstruct.2013.06.038

Pérez, C., Collazo, A., Izquierdo, M., Merino, P., \& Nóvoa, X. R. (2002). Comparative study between galvanised steel and three duplex systems submitted to a weathering cyclic test. Corrosion Science, 44(3), 481-500. Recuperado de https:// doi.org/10.1016/S0010-938X(01)00070-1

Rani, B. E. A., \& Basu, B. B. J. (2011). Green Inhibitors for Corrosion Protection of Metals and Alloys: An Overview. International Journal of Corrosion, 2012 https://doi. org/10.1155/2012/380217

Romero, C. (2014). Determinación del comportamiento ante el desgaste por frotamiento del acero dúplex SAF 2507 usando un diseño experimental Taguchi. Revista Ingeniería UC, Vol. 21, No. 2, 16-26, Agosto 2014. Recuperado de https:// www.redalyc.org/pdf/707/70732656003.pdf

Schmiedl Briceño, E. C., \& Audelo Guevara, F. J. (2017). Diseño y construcción de una cámara de niebla salina mediante la norma ASTM B-117 para ensayos de corrosión para la carrera de Ingeniería Química de la ESPOCH. (tesis de grado). Escuela Superior Politécnica de Chimborazo, Riobamba. Recuperado de http://dspace.espoch.edu.ec/ handle/123456789/6529

Valenzuela Guerrero, S. P. (2016). Influencia de ácidos húmicos en la corrosión de zinc puro para simular la corrosión de revestimientos $H D-Z N$ en el suelo. (tesis de grado). Universidade Federal do Rio Grande do Sul. Escola de Engenharia. Curso de Engenharia Metalúrgica. Recuperado de https://www.lume.ufrgs.br/handle/10183/149804

Vera, R.M., \& Cañas, F.J. (2005). Corrosion Behaviour of Galvanized and Coated Galvanized Steel (Duplex) in Marine Environment. Información tecnológica, 16(4), 5358. https://dx.doi.org/10.4067/S0718-07642005000400009

Vilche, J. R., Bucharsky, E. C., \& Giúdice, C.A. (2002). Application of EIS and SEM to evaluate the influence of pigment shape and content in ZRP formulations on the corrosion prevention of naval steel. Corrosion Science, 44(6), 1287-1309. https:// doi.org/10.1016/S0010-938X(01)00144-5

Worsley, D. A., Williams, D., \& Ling, J. S. G. (2001). Mechanistic changes in cut-edge corrosion induced by variation of organic coating porosity. Corrosion Science, 43(12), 2335 2348. https://doi.org/10.1016/S0010-938X(01)00026-9 